\title{
Guidelines for telematic second opinion consultation on headaches in Europe: on behalf of the European Headache Federation (EHF)
}

\author{
José Pereira-Monteiro • Maria-Magdalena Wysocka-Bakowska • \\ Zaza Katsarava · Fabio Antonaci
}

Received: 7 February 2010/Accepted: 25 March 2010/Published online: 7 April 2010

(C) Springer-Verlag 2010

\begin{abstract}
The seeking of a second opinion is the longestablished process whereby a physician or expert from the same or a similar specialty is invited to assess a clinical case in order to confirm or reject a diagnosis or treatment plan. Seeking a second opinion has become more common in recent years, and the trend is associated with significant changes in the patient-doctor relationship. Telemedicine is attractive because it is not only fast but also affordable and thus makes it possible to reach highly qualified centres and experts that would otherwise be inaccessible, being impossible, or too expensive, to reach by any surface transport. In Europe, the European Headache Federation (EHF), being able to draw on a group of headache experts covering all the European languages, is the organisation best placed to provide qualified second-opinion consultation on difficult headache cases and to develop a Headache
\end{abstract}

Electronic supplementary material The online version of this article (doi:10.1007/s10194-010-0211-6) contains supplementary material, which is available to authorized users.

J. Pereira-Monteiro $(\square)$

Department of Neurology, Hospital Santo António and ICBAS,

University of Porto, Porto, Portugal

e-mail: pereiramonteiro@mail.telepac.pt

M.-M. Wysocka-Bakowska

Headache and Pain Policlinic, Warsaw, Poland

Z. Katsarava

Department of Neurology, University of Essen,

Hufelandstrass 55, 45122 Essen, Germany

F. Antonaci

University Centre for Adaptive Disorders and Headache

(UCADH), Pavia, Italy

F. Antonaci

Headache Medicine Centre, Policlinic of Monza, Monza, Italy
Medical Opinion Service Centre. The provision of good quality clinical information is crucial to the formulation of a valid, expert second opinion. This preliminary step can be properly accomplished only by the primary health care provider through the furnishing of an appropriate clinical report, together with the results of all available tests, including original films of all imaging studies already performed. On receiving the EHF's proposed standardised data collection form, properly filled in, we may be sure that we have all the relevant data necessary to formulate a valid expert second opinion. This form can be accessed electronically and downloaded from the EHF website. Once finalised, the EHF second opinion project should be treated as a pilot strategy that requires careful monitoring (for the first year at least), so that appropriate changes, as suggested by the retrospective analysis and its quality control, can be implemented.

Keywords Headaches - Guidelines - Telemedicine · Telematic $\cdot$ Second opinion $\cdot$ Consultation

\section{Introduction}

The seeking of a second opinion is the long-established process whereby an expert from the same or a similar specialty is invited to assess a clinical case in order to confirm or reject a diagnosis or treatment plan. A second opinion might be requested by the primary physician (primary health care provider, PCP), by the patient, or by the patient's relatives. It serves to reduce uncertainty and thus anxiety, and to promote a better understanding of the disease by the patient and her/his family, as well as better compliance with the treatment plan. When a second opinion confirms the initial diagnosis, it may indeed provide 
reassurance and help the patient to accept her/his disease [1].

By bridging an important gap between the primary physician's diagnosis and treatment plan and the patient's emotional need for expert opinion, a medical second opinion should thus help to establish a patient's medical needs and contribute to achieving optimal treatment goals.

Because traditional second opinion evaluations involve a face-to-face examination, they can be difficult to obtain, rather expensive and involve a delay; as a result, they tend to be carried out in very few or very special cases.

However, in recent decades, advances in the field of information technology, and in telecommunications technology in particular, have led to the development of a new model of second opinion and the creation of the concept of telemedicine.

Nowadays, with access to the internet so widespread, patients and doctors are directly involved in establishing primary diagnoses and in the treatment decision-making process. Both categories actively use the internet to get information about a disease and about modern treatment options and strategies and will often actively seek second opinions from opinion leaders in the field.

Initially, telemedicine (the use of telecommunications technology for medical diagnosis and patient care) was mainly used for getting second opinions on imaging data (e.g. radiological and neuroradiological images, online ECG, USG, etc.). However, this novel concept rapidly spread to other medical specialties: pathology, surgery, cardiology, dermatology, orthopaedics, gynaecology, urology, neurology, including neurosurgery and so on [2-4].

Telemedicine [5] has proved attractive because it is not only fast but also affordable and thus makes it possible to reach highly qualified centres and experts that would otherwise be inaccessible (unavailable, impossible or too expensive to reach by any surface transport) $[2,5,6]$.

As a result of this trend, which is one of the reasons why second opinion seeking has become more common in recent years, the relationship between patients and doctors has changed radically: the era of the paternalistic relationship, in which patients blindly followed the advice of their doctors, is over.

\section{The European Headache Federation project}

In the field of headache medicine in Europe, the European Headache Federation (EHF), being able to draw upon the expertise of a group headache experts covering all the European languages, is the organisation best placed to provide qualified second opinion consultation on difficult headache cases. Through its development of a Headache Medical Opinion Service Centre, the EHF will provide a valuable and necessary service to the populations and health care providers of all European countries, while also accomplishing the most important of its aims: to promote headache knowledge and care in Europe [7-9].

The provision of good quality clinical information is crucial to the formulation of a valid, expert second opinion. This preliminary step can be properly accomplished only by the PCP through the furnishing of an appropriate clinical report, together with the results of all available tests, including the original films of all imaging studies already performed.

Furthermore, it may be better accomplished if a standard data collection form is provided in advance. To this end, several second opinion software solutions have been developed to facilitate communication and prevent the omission of important, sensitive data, while guaranteeing adequate personal data protection [6].

To the best of our knowledge, no such software programs are available for the requesting and formulation of second opinions on headache patients, even though there is a need for them in order to guarantee optimal results. When assessing a difficult case, there are certain crucial data that must always be collected. First of all, it is important to know the reason for the consultation: whether it is to confirm a diagnosis, for differential diagnosis or diagnostic work-up counselling, or for treatment advice. The need for treatment advice may arise when a patient fails to respond to a therapy or develops side effects, or it may simply stem from a desire to explore all possible and available therapeutic options in order to optimise a patient's treatment.

The expert should also know who is requesting the second opinion: whether it is the patient her/himself, family members and/or friends, or a doctor (i.e., the PCP). If it is the PCP, it is important to know his/her name, affiliation and contact details in case he/she needs to be contacted again in order to get more detailed clinical information.

The best way to provide the necessary information is through a semi-structured questionnaire. The data collection form (available as Electronic Supplementary Material) must cover a series of important aspects, as detailed in the following steps:

1. Patient identification (name/initials, gender, birth date/ age, country/nationality, language);

2. A brief present disease (headache) history including

a. Headache characteristics (type 1,2,3 or more), date of onset, location (unilateral, side shifting, bilateral), quality of pain (pulsating, tightening, stabbing), whether headache worsens with physical activity, the presence of aura symptoms (visual, sensory, motor) and associated symptoms 
(phonophobia, photophobia, nausea or vomiting), as well as dysautonomic symptoms (red eye, tearing, rhinorrhoea, pacing around) or basilar artery symptoms (diplopia, vertigo, tinnitus, hypoacusia, ataxia), or any others.

b. It is also important to indicate the temporal pattern of the headache using the temporal definitions given in the IHS criteria [10] (acute/episodic; subacute/progressive; chronic paroxysmal or continuous), as this aspect may be difficult to establish without a clinician's contribution.

3. Details of the patient's previous medical history, particularly with regard to comorbid psychiatric and internal diseases and the medications used to treat them. This information is very important, and should always be available.

4. Family medical history, as well as social and professional background and habits. This information, too, can be highly relevant.

5. A full clinical examination. It is crucial to have an overview of the patient's clinical conditions, including the results of general and neurological examinations (mental status, cranial nerves, motor system, reflexes, sensory and cerebellar system evaluation), but the quality of this overview will depend on the quality of the clinician information provided.

6. Results of diagnostic work up, namely of

- Blood laboratory tests: haematology, biochemistry, immunology and serology, (ESR, CRP);

- Neurophysiological examinations (EEG, EMG or EP) and echo-Doppler, USG, transcranial-Doppler of extracranial (carotid, vertebral) and intracranial vessels,

- Neuroimaging studies (CT, MRI, or ANGIO MRI), when advisable and available.

- All other evaluations, including, for example, cardiological, ophthalmological and ENT consultations.

It is also important to know the suggested clinical diagnosis (headache type or types), as formulated by the PCP, as well as the patient's other current comorbid medical conditions.

Information should be given regarding current headache treatments (prescribed and over-the-counter medications) for acute and for prophylactic therapy (drugs, doses, treatment duration, response and side effects). Use/overuse of OTC drugs and/or other substance use or abuse should be reported, as should treatments used for other comorbidities [8].

On receiving this proposed standardised form, properly completed, we can be sure that all the relevant data have been provided, and a better result, in terms of a valid expert second opinion, may thus be expected [11, 12]. The proposed application form can be accessed electronically and downloaded from the EHF website, automatically translated into the user's language, thereby facilitating communication [8, 9, 11, 12].

The authors of the present document can be contacted for consultations on behalf of the EHF, but other experts in the field are also welcome to compose a list of opinion leaders on the field (Board of Headache European Consultants) that can provide the requested second opinion, If possible, with the same language of the informer, with quality and safety using appropriate telecommunicating technology [11] (i.e. telecommunication or Skype connection).

Through this project, the EHF will open the way for better care for patients with difficult or rare headache conditions, and also for people from remote and/or small places where health care facilities are more restricted and gaining access to a headache expert can be difficult.

Through modern telecommunications technology and internet teleconsultation, the EHF might thus be enabled to accomplish, on a global level, its mission to provide strong medical expert support in the field of headache.

The objectives the EHF aims to achieve through the implementation of this service are:

1. Secure and fast access to patient information, wherever the patient is located, also making use of on-line dialogue methods (i.e. Skype);

2. better quality diagnosing and treatment;

3. reduced time to treatment;

4. reduced use of OTC drugs;

5. promotion of more efficient use of resources; and

6. promotion of on-line collaboration among health care professionals (across health care organisations and national borders).

Once finalised, the programme should be treated as a pilot strategy and be carefully monitored (for the first year at least), so that appropriate changes, as suggested by the retrospective analysis and its quality control, can be implemented.

The main problem this project could encounter, in the event of a large volume of requests, is that of the costs involved. It is important to estimate these, i.e., the total cost of implementing the project, as well as the costs per case. It is also essential to establish how these costs will be met (who will pay) and to secure the funds needed. The success of the project will also depend on the availability of a good programme, software and informatics with a broadly available platform.

Therefore it is necessary to consider all the possible sources of funding, asking as to whom we should look to for the necessary grants and financial support: the EHF, the 
WHO, local health authorities, the pharmaceutical industry, or private companies.

Since the EHF is a non-profit organisation, which seeks to accomplish a mission and pursues prestige rather than financial gain, this reduces the total costs of the programme and should make it possible to obtain financial support from an external sponsor.

A further consideration is the legal question of the liability and responsibility of the consulting experts offering diagnoses and proposing treatments. The expert second opinion they provide should be presented and considered purely as advice, making it quite clear that full responsibility cannot be accepted for advice given on the basis of information provided by a primary health physician. This issue, however, needs further discussion and clarification.

Conflict of interest None.

\section{References}

1. Second opinion of the European Data Protection Supervisor on the review of Directive 2002/58/EC concerning the processing of personal data and the protection of privacy in the electronic communications sector (directive on privacy and electronic communications) (2009/C 128/04) Web

2. Chronaki CE, Zabulis X, Katehakis DG, Giannopoulos A, Stathiakis N, Tsiknakis M, Lees PJ, Simantirakis EN, Vardas PE, Orphanoudakis SC (1998) WebOnCOLL enabled remote cardiology consultation for suspected myocardial infarction. In: Proceedings of Mednet'98, London, England, 16-19:41-43
3. Bourne J, Harris D, Mayadas F (2005) Online engineering education: learning anywhere, anytime. J Asynchronous Learn Netw 9(1): $1-28$

4. Gordon C (1995) Practice guidelines and healthcare telematics: towards an alliance. In: Gordon C, Christensen JP (eds) Health telematics for clinical guidelines and protocols, IOS Press

5. Orphanoudakis SC, Chronaki C, Kostomanolakis S (1994) I2C:a system for the indexing, storage, and retrieval of medical images by content. Med Inform Lond 19(2):109-122

6. Chronaki CE, Katehakis DG, Zabulis XC, Tsiknakis M, Orphanoudakis SC (1997) WebOnCOLL: medical collaboration in regional healthcare networks. IEEE Trans Inf Technol Biomed 1(4):257-269

7. Gerber WD, Pfaffenrath V (1993) Headache in Europe. In: Gerber WD, Pfaffenrath (eds) Arcis Verlag GmbH, Munchen $5: 67-72$

8. Antonaci F, Láinez JM, Diener HC, Couturier EG, Agosti R, Afra J, Färkkilä M, Obelieniene D, Valade D (2005) Guidelines for the organization of headache education in Europe: the headache school; European Headache Federation. Funct Neurol 20(2): 89-93

9. Antonaci F, Valade D, Lanteri-Minet M, Láinez JM, Jensen R, Steiner TJ (2008) Proposals for the organization of headache services in Europe; European Headache Federation and lifting the burden: the global campaign to reduce the burden of headache worldwide. Intern Emerg Med 3(Suppl 1):S25-S28

10. Headache classification committee of the International Headache Society (2004) The international classification of headache disorders, 2nd edn. Cephalalgia 24(Suppl 1):9-160

11. Medina Mores R, Winograd T, Flores F, Flores M (1993) The action workflow approach to workflow management technology. Inf Soc 9:391-406

12. Scalvini S, Zanelli E, Martinelli G, Baratti D, Giordano A, Glisenti $F$ (2005) Cardiac event recording yields more diagnoses than 24-hour holter monitoring in patients with palpitations. J Telemed Telecare 11(Suppl):14-16 